\title{
Castrate Resistant Metastatic Prostate Cancer: Options for a Patient in 2012
}

\author{
Asim Ahmad ${ }^{1}$, Winston Tan ${ }^{2 *}$ \\ ${ }^{1}$ Internal Medicine Mayo Clinic Florida, Jacksonville, USA \\ ${ }^{2}$ Division of Hematology, Oncology Mayo Clinic Florida, Jacksonville, USA \\ Email: asim@mayo.edu, ${ }^{*}$ winston@mayo.edu
}

Received August 6, 2012; revised September 11, 2012; accepted September 28, 2012

\begin{abstract}
Castrate resistant metastatic prostate cancer remains a fatal disease. Through preclinical studies and clinical trials, a multitude of options have been made available to prolong the life of these patients, as well as improve quality of life. First line treatment options following tumor progression after androgen deprivation therapy include Provenge and docetaxel. Several options are available as second line treatment, including cabazitaxel, abiraterone, and enzalutamide. Many drugs currently being studied are very promising, such as alpharadin. Here we review treatment options for patients suffering from disease progression after androgen deprivation therapy, and offer a review of the current available options for the clinician.
\end{abstract}

Keywords: Castrate; Resistant; Prostate; Cancer; Treatment; Review

\section{Introduction}

Prostate cancer is the most common cancer for men in the US with approximately 200,000 new cases diagnosed yearly [1]. It is also the second leading cause of cancer deaths accounting for 28,471 deaths [2]. If diagnosed at an early stage, prostate cancer is a highly curable disease. Five year relative survival rates were $100.0 \%$ for both localized and regional disease, and $27.8 \%$ for distant disease [3]. Despite having a favorable prognosis, there is a subgroup of prostate cancer patients who develop incurable metastatic disease.

Initial standard treatment for metastatic prostate cancer is androgen-deprivation therapy, either through medical or surgical castration. Initially, metastatic prostate cancer relies on androgen for growth in the majority of cases. Most androgen is produced by the testes and is regulated by the hypothalamic-pituitary access. Although androgen-deprivation therapies typically result in significant Prostate Specific Antigen (PSA) responses, nearly all patients develop a progressive castration-resistant disease. This is defined as disease progression despite therapy and may include increasing PSA, new clinical metastasis, or progression of existing metastasis. Clinically it may present as fatigue, bone pain, malaise, or a myriad of other nonspecific symptoms.

Several treatment options have been described for patients with castration-resistant prostate cancer (CRPC);

${ }^{*}$ Corresponding author. however, most of the secondary hormone manipulation techniques, despite achieving castrate levels of testosterone, only temporarily control disease without improvement in overall survival; average median survival remains around 30 months. On average, after 12 - 18 months, the cancer develops resistance to therapy [4].

Analysis of tumor specimens from patients with CRPC have shown several mechanisms adapted by cancer cells to reactivate the androgen receptor (AR) signaling at sub-physiological serum concentrations of androgens, even in the presence of AR antagonists. These include mutations of the AR and changes in conformation of the binding site [5]. Several new drugs targeting these pathways have been developed (Table 1). The following is a review regarding recent developments in the treatment of advanced CRPC.

\section{Methods}

We performed a systematic review of peer-reviewed publications identified through searches of MEDLINE/ PubMed from 2003 to September 2012. We also included results of the relevant clinical trials presented at annual oncology meetings. The ongoing phase II and phase III trials for first and second-line chemotherapy agents for CRPC were reviewed from the US National Institute of Health's web resource and clinicaltrials.gov, which is a registry of clinical trials conducted in the United States and worldwide. 
Table 1. First and second line drugs indicated for CRPC, their adverse effects, mean overall survival benefit, and contraindications.

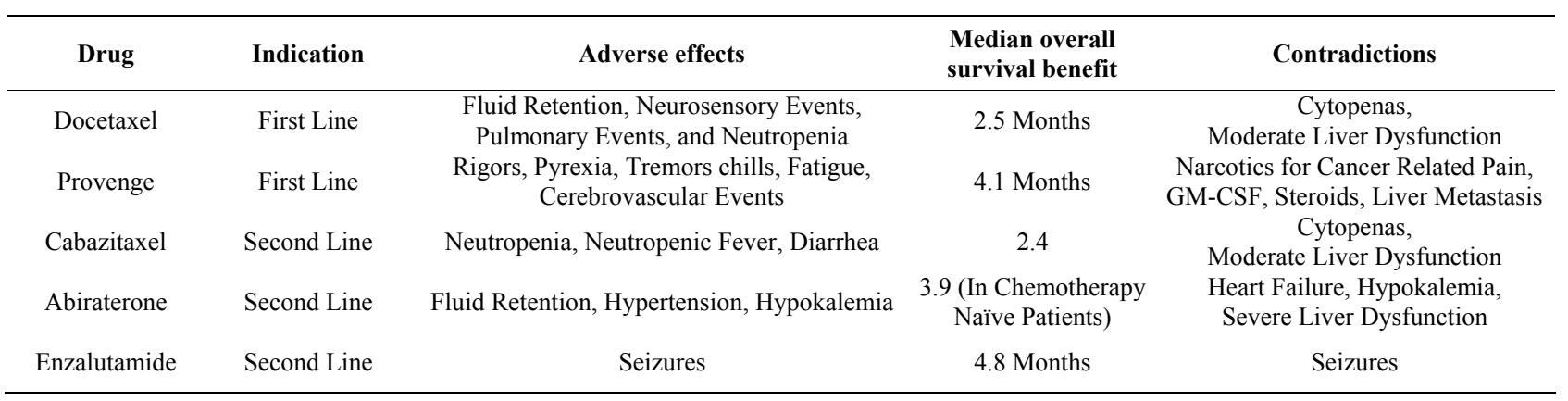

\section{Results/Discussion}

\subsection{First Line Treatment Options for Castrate Resistant Metastatic Prostate Cancer}

Docetaxel remains standard first line treatment of CRPC. The TAX-327 trial and the Southwest Oncology Group (SWOG) 99-16 study both showed survival benefits when compared to other chemotherapy agents (mitoxantrone based).

Docetaxel promotes the assembly of microtubules and inhibits the depolymerization of tubulin, stabilizing microtubules in the cell. This results in the inhibition of DNA, RNA, and protein synthesis. In the TAX-327 trial, 1006 chemotherapy-naïve CRPC patients were randomized to three different treatment arms: docetaxel every week, docetaxel every three weeks, or mitoxantrone every three weeks. All three groups received prednisone. Patients receiving docetaxel every three weeks had a significant improvement of survival compared to weekly docetaxel and mitoxantrone (18.9 months vs 16.5 months). An update of the results of TAX-327 trial in 2007 showed a persistence of survival benefit for docetaxel every three weeks compared to mitoxantrone. At three years, survival was $17.2 \%$ for docetaxel every three weeks compared to $12.8 \%$ with mitoxantrone [6]. In the Southwest Oncology Group (SWOG) 99-16 study, 674 patients with metastatic CRPC were randomized to docetaxel/estramustine and mitoxantrone/prednisone. Docetaxel was reported to be superior to mitoxantrone with a median survival of 17.5 months versus 15.6 months and PSA declines of $50 \%$. These two trials showed a $20 \%-24 \%$ reduction in mortality in patients with CRPC receiving docetaxel chemotherapy doublets. Common adverse events noted in these trials include: fluid retention, neurosensory events, pulmonary events, and neutropenia.

Provenge (sipuleucel-T) is an autologous cellular immunotherapy agent which stimulates an immune response against prostate cancer. It is approved as first line therapy for asymptomatic or minimally symptomatic metastatic castrate resistant prostate cancer patients. Provenge is designed to stimulate T-cell immunity against prostatic acid phosphatase, an antigen found in large amounts of prostate cancer tissue, but not in non-prostate cancer tissue. The study NCT01133704, a phase III, placebo controlled trial, was undertaken to evaluate efficacy of this agent [7]. A total of 127 patients with asymptomatic, metastatic CRPC were randomly assigned to receive three infusions of Provenge (82 patients) or placebo (45 patients) every 2 weeks. The median time to disease progression for Provenge was 11.7 weeks compared with 10.0 weeks for placebo. Median survival was 25.9 months with Provenge and 21.4 months for placebo. Provenge was generally well tolerated with most toxicities being grade 1 or 2, which included rigors, pyrexia, and tremors. The study concluded that though Provenge fell short of demonstrating a statistically significant difference in time to disease progression, its primary endpoint, it may provide a survival advantage to asymptomatic CRPC patients.

In study NCT00065442, a double-blind, placebo-controlled, multicenter phase III trial, 512 patients, either asymptomatic or mildly symptomatic, were randomly assigned to receive either Provenge (341 patients) or placebo (171 patients) every 2 weeks for a total of three infusions [8]. The primary end point was overall survival. In the Provenge group, there was a relative reduction of $22 \%$ in the risk of death compared to the placebo group. This reduction (25 vs 22 months) represented a 4.1month improvement in median survival. The time to objective disease progression was similar in the two study groups. Among patients with PSA assessments after baseline, reductions of at least $50 \%$ on two visits at least 4 weeks apart were observed in 8 of 311 patients $(2.6 \%)$ in the Provenge group, as compared with 2 of 153 patients $(1.3 \%)$ in the placebo group.

A combined analysis of these two trials as well as a third randomized control trial found the following adverse events were more common with Provenge than with placebo: chills (53\% versus $11 \%)$ and fatigue $(41 \%$ versus $35 \%$ ). Cerebrovascular events were reported in $3.5 \%$ of patients treated with Provenge and $2.4 \%$ of patients who received placebo $[9,10]$. 


\subsection{Second Line Treatment of Castrate Resistant Metastatic Prostate Cancer}

Despite being "hormone refractory", numerous preclinical and clinical studies have shown that prostate cancer cells have persistent high androgen receptor expression and thus mediate androgen signaling [11].

Cabazitaxel was the first drug approved by the Food and Drug Administration (FDA) for CRPC in patients whose disease had progressed during or after docetaxel therapy. Cabazitaxel is a tubulin-binding taxane drug that in preliminary studies had been shown to confer antitumor activity in models resistant to docetaxel.

Study NCT00417079 (Tropics trial), a randomized phase III trial, allocated 755 men with metastatic CRPC whose disease had progressed despite hormonal therapy, and a chemotherapy regimen including docetaxel into two randomly assigned groups [12]. One group would receive mitoxantrone (377 patients) intravenously and the other cabazitaxel (378 patients) intravenously every 3 weeks, both with prednisone. Final analysis revealed median survival was 15.1 months in the cabazitaxel group and 12.7 months in the mitoxantrone group. Median progression-free survival, as measured by PSA progression, tumor or pain progression, or death, was 2.8 months in the cabazitaxel group and 1.4 months in the mitoxantrone group. PSA progression in the cabazitaxel arm occurred at 6.4 months and 3.1 in the mitoxantrone group. Tumor progression occurred in 5.4 months in the mitoxantrone group and 8.8 months in the cabazitaxel group. Neutropenia was the most common grade three or higher adverse event occurring in 303 patients in the cabazitaxel arm, with neutropenic fever developing in 28 patients, and 215 patients in the mitoxantrone arm, with neutropenic fever developing in 5 patients. Diarrhea was also noted, occurring in 28 patients being administered cabazitaxel and 5 being administered mitoxantrone.

A phase III clinical trial, study NCT01308567, is currently active and scheduled to be completed in December of 2017. It will measure efficacy of cabazitaxel versus docetaxel in regards to first line treatment of metastatic CRPC [13].

Several studies have indicated that effective treatment of CRPC could be obtained by either further suppressing or maximally suppressing testosterone production. Numerous drugs are currently in development to achieve this aim. Drugs currently FDA approved that utilize this mechanism to treat CRPC include abiraterone and enzalutamide.

Abiraterone acetate is a highly selective irreversible inhibitor of cytochrome P-17, a dual enzyme that blocks androgen production that is expressed in testicular, adrenal, and prostatic tumor tissues. It inhibits production of testosterone precursors androstenedione and dehydroepi- androsterone [14]. In a phase II, multicenter study of 58 patients who had progressive, metastatic CRPC and failed hormonal therapy of up to two cytotoxic regimens, one of which must have been docetaxel, were treated with abiraterone and prednisone [15]. Twenty-five of 56 patients $(45 \%)$ had a PSA decline of $50 \%$ or more. PSA progression, as defined by Prostate Specific Antigen Working Group 1 criteria, occurred at a median time of 169 days. The majority of abiraterone-related adverse events were grades 1 - 2 and no grade 4 adverse events were reported. Also noted was a significantly better PSA response in the ketoconazole-naïve (another inhibitor of cytochrome P-17), post-docetaxel CRPC population.

The first phase III trial studying abiraterone, whose primary end point was overall survival, was terminated early as interim analysis exceeded prespecified criteria [16]. In this study, 1195 men were randomly assigned in a 2:1 ratio to either abiraterone or placebo both with prednisone groups. After a median follow-up of 13 months, treatment with abiraterone increased overall survival compared to placebo, median 14.8 versus 10.9 months. Statistically significant improvements were also seen in time to PSA progression (defined as a $25 \%$ increase over the baseline and an increase in the absolute-value PSA level by at least $5 \mathrm{ng}$ per milliliter) at 10.2 versus 6.6 months, progression-free survival 5.6 versus 3.6 months, and PSA response rate, $29 \%$ versus $6 \%$ in the abiraterone group when compared to placebo. Mineralocorticoid-related adverse events, including fluid retention, hypertension, and hypokalemia, were more frequently reported in the abiraterone group than in the placebo.

Preliminary results regarding abiraterone and symptomatic bone metastasis were presented at ASCO (American Society of Clinical Oncology) 2011 [17]. Highlights include a statistically significant improvement in palliation of pain due to bone metastases in patients treated with abiraterone compared with placebo. Median duration of improvement was 10.3 versus 5.6 months, and median time to progression in pain intensity, 7.4 versus 4.7 months. Also, there was a statistically significant increase in the time to first skeletal-related event, defined as pathologic fracture, spinal cord compression, or palliative radiation therapy or surgery, median 301 versus 150 days.

One of latest drugs to be approved by the FDA for treatment of CRPC is enzalutamide; receiving approval on August 31, 2012, and scheduled to be available to patients by mid-September 2012 .

Enzalutamide exploits CRPC tendency to over express androgen receptors by inhibiting nuclear translocation of the androgen receptor, DNA binding, and co-activator recruitment. AFFIRM was an international, phase III, randomized, double-blind, placebo-controlled study of 
enzalutamide in patients with prostate cancer who had previously been treated with one or two chemotherapy regimens, at least one of which contained docetaxel [18]. A total of 1199 patients with CRPC were randomized to receive either enzalutamide (800 patients) or placebo (399 patients). The primary endpoint of the trial was overall survival. Enzalutamide-treated patients had a statistically significant improvement in median overall survival compared to the placebo group (18.4 versus 13.6 months). Enzalutamide provided a $37 \%$ reduction in risk of death compared to placebo. PSA progression, defined as an increase by a factor of 1.25 over the baseline level, was 8.3 months in the treatment arm versus 3 months in the control arm. Seizure was noted to occur in $0.9 \%$ of patients on enzalutamide and none of the placebo-treated patients. Grade 3 and higher adverse reactions were reported among $47 \%$ of enzalutamide-treated patients compared to $53 \%$ in the placebo arm.

The role of abiraterone and enzalutamide in chemotherapy naïve patients is currently under investigation. The results of studies to evaluate efficacy are currently awaiting longer follow-up data. Clinicians have already been using abiraterone off label in chemotherapy naïve patients. Data presented at ASCO 2012 regarding results from an interim analysis of a phase III clinical trial involving 1088 men with metastatic, asymptomatic or minimal symptomatic castration resistant prostate cancer, who did not receive prior chemotherapy, that were randomly assigned to abiraterone plus prednisone or placebo plus prednisone demonstrated increased radiographicprogression free survival. Median was not reached in the abiraterone group versus 8.3 months in the placebo group [19]. This interim analysis also demonstrated overall survival in the abiraterone group, median not reached versus 27.2 months in the placebo group. As a caveat, overall survival data is confounded by the use of additional active therapy following trial participation.

\subsection{Treatment Options for Bony Metastasis}

Bone metastasis is a common occurrence in patients with CRPC. Numerous modalities have been evaluated in preclinical and clinical trials in an attempt to find agents that can slow disease progression and offer symptomatic relief. Two of the best studied agents are zolendronic acid and denosumab. One of the end points utilized in most of these trials to evaluate these drugs was first skeletal-related event, which as defined above includes the time until pathologic fracture, spinal cord compression, or palliative radiation therapy or surgery.

Zoledronic acid, a bisphosphonate, has been shown to delay progression of skeletal related events and also blunt the bone loss associated with androgen deprivation therapy. In a randomized, placebo-controlled trial, patients with hormone-refractory prostate cancer and a his- tory of bone metastases were assigned to a regimen of intravenous zoledronic acid at $4 \mathrm{mg}$ (214 patients), zoledronic acid at $8 \mathrm{mg}$ and then subsequently reduced to 4 $\mathrm{mg}$, secondary to renal toxicity $(8 / 4 \mathrm{mg}$ ) (221 patients), or placebo (208 patients) every 3 weeks for 15 months [20]. Endpoints included proportions of patients with skeletal-related events, disease progression, and safety. A greater proportion of patients who received placebo had skeletal-related events than those who received zoledronic acid at $4 \mathrm{mg}(44.2 \%$ versus $33.2 \%)$ or those who received zoledronic acid at $8 / 4 \mathrm{mg}(38.5 \%$ versus placebo). There were no statistically significant differences between patients who received zoledronic acid and those who received placebo regarding the percent change from baseline serum PSA within 30 days of progression of disease, implying that zoledronic acid had no substantial effect on the clearance, secretion, or measurement of PSA. The median time of survival was 464 days for patients who received placebo, 546 days for patients who received zoledronic acid at $4 \mathrm{mg}$, and 407 days for patients who received zoledronic acid at $8 / 4 \mathrm{mg}$. The most common adverse events that occurred during the trial included fatigue, anemia, myalgia, fever, and lower-limb edema. They occurred in at least $5 \%$ more for the patient population in both zoledronic acid groups compared to the placebo group. A more recent placebo controlled trial echoed these results [21] finding that in a trial of zoledronic acid (214 patients) versus placebo (208 patients), zoledronic acid led to significantly reduced skeletal pain.

Denosumab is a monocloncal antibody that binds to RANK ligand, inhibiting osteoclast formation and activation. It is indicated for the prevention of skeletal related events in men with prostatic metastasis to the bone and for the treatment of bone loss in men receiving androgen depravation therapy. A double-blind, phase III trial involving 1901 men with CRPC and at least one bone metastasis were randomly assigned to denosumab or zoledronic acid [22]. The primary objective of the study was time to first skeletal-related event. First skeletal-related event was delayed with denosumab versus zoledronic acid, median 20.7 months versus 17.1 months. There was no statistically significant difference in either overall survival or time to disease progression. PSA was measured every three months and remained similar in both treatment groups. Both treatments were well tolerated. Osteonecrosis of the jaw was more prevalent with denosumab compared to zoledronic acid. Hypocalcemia was also more frequent with denosumab $(13 \%$ versus $6 \%)$.

In another phase III, double-blind, randomized, placebo-controlled study, NCT00286091, men with non-metastatic CRPC at high risk of bone metastasis, defined as a PSA $\geq 8.0 \mu \mathrm{g} / \mathrm{L}$ or PSA doubling time $\leq 10.0$ months, or both, were enrolled [23]. Patients were randomly as- 
signed to receive subcutaneous denosumab (716 patients) or subcutaneous placebo (716 patients) every 4 weeks. The primary endpoint was bone-metastasis-free survival, determined by either first occurrence of bone metastasis (symptomatic or asymptomatic) or death from any cause. Denosumab significantly increased bone-metastasis-free survival by a median of 4.2 months compared with placebo. Denosumab also significantly delayed time to first bone metastasis (33.2 versus 29.5 months). Overall survival did not differ between the groups. Median PSA at baseline and change from baseline were also similar in both groups. Rates of adverse events and serious adverse events were similar in both groups, except for osteonecrosis of the jaw and hypocalcaemia, which occurred more frequently in the denosumab group.

Radium-223 (223Ra) is an alpha particle emitting radiopharmaceutical. It is a promising new drug that is currently seeking FDA approval. Radium is a boneseeking element, and its decay allows the deposition of high energy radiation over a shorter distance than with beta particle emitting radioisotopes. Theoretically, this allows for the treatment of tumor metastasis while minimizing toxicity to the bone. 223Ra increased overall survival and reduced the incidence of serious skeletal-related events in the phase III ALSYMPCA trial [24]. In this trial, all patients had CRPC with multiple bone metastases and had either progressed on docetaxel chemotherapy or were not candidates for docetaxel chemotherapy. Patients were required to have two or more bone metastases and no known visceral metastases. Overall, 921 patients were randomly assigned in a 2:1 ratio to 223Ra or placebo. Updated results were presented at the 2012 ASCO meeting, based upon the entire study population of 921 patients; overall survival was significantly prolonged with 223Ra compared to placebo, median 14.9 versus 11.3 months. The time to first skeletal-related event was extended, median 12.2 versus 6.7 months. Treatment was well tolerated. There were only minimal increases in myelosuppression compared with placebo (grade 3 or 4 neutropenia, $1.8 \%$ versus $0.8 \%$, and grade 3 or 4 thrombocytopenia, $6 \%$ versus $2 \%$ ).

In a placebo-controlled, phase 2 study, patients with hormone-refractory prostate cancer and bone pain needing external-beam radiotherapy were assigned to four intravenous injections of 223Ra (33 patients) or placebo (31 patients), given every 4 weeks [25]. Primary endpoints included change in bone-alkaline phosphatase (ALP) concentration, a marker of skeletal metastasis. Median relative change in bone ALP during treatment was $-65.6 \%$ and $9.3 \%$ in the $223 \mathrm{Ra}$ group and placebo groups respectively. Secondary endpoints, included time to PSA progression. Median relative change in PSA from baseline to 4 weeks after last study injection was $-23.8 \%$ in the $223 \mathrm{Ra}$ group and $44.9 \%$ in the placebo group. A confirmed PSA response of more than 50\% was seen in 11 of 31 patients assigned to $223 \mathrm{Ra}$ and 5 of 28 assigned to placebo.

\section{Conclusions}

As reviewed, research in men with CRPC has lead to a variety of drug therapies which provide symptomatic control, delay disease progression, and increase overall survival. The proper sequence of administration will vary and is likely to be based on a host of factors including extent of disease involvement, sites of disease, as well as rate of disease progression. In addition, cost, side effect profile, patient preference, and insurance reimbursement may also influence a clinician to pursue a certain drug regimen.

Furthermore, information gleaned in future clinical trials as well as through genomic and proteomic testing will hopefully allow the clinician to identify subsets of patients that would benefit from certain treatments and sequence drugs appropriately.

\section{REFERENCES}

[1] "Prostate Cancer Statistics," 2012. http://www.cdc.gov/cancer/prostate/statistics

[2] V. T. DeVita, T. S. Lawrence and S. A. Rosenberg, "Cancer Principles and Practice of Oncology," 2008.

[3] N. Howlader, "SEER Stat Fact Sheets: Prostate," 2012. http://seer.cancer.gov/statfacts/html/prost.html

[4] K. Lassi and N. A. Dawson, "Emerging Therapies in Castrate-Resistant Prostate Cancer," Current Opinion in Oncology, Vol. 21, No. 3, 2009, pp. 260-265. doi:10.1097/CCO.0b013e32832a 1868

[5] R. M. Attar, C. H. Takimoto and M. M. Gottardis, "Castration-Resistant Prostate Cancer: Locking up the Molecular Escape Routes," Clinical Cancer Research, Vol. 15 , No. 10, 2009, pp. 3251-3255. doi:10.1158/1078-0432.CCR-08-1171

[6] D. R. Berthold, G. Pond, R. de Wit, et al., "Docetaxel plus Prednisone or Mitoxantrone Plus Prednisone for Advanced Prostate Cancer: Updated Survival of Tax 327," Journal of Clinical Oncology, Vol. 25, No. 18, 2007, pp. 242-245.

[7] E. J. Small, et al., "Placebo-Controlled Phase III Trial of Immunologic Therapy," Journal of Clinical Oncology, Vol. 24, No. 19, 2006, pp. 3089-3093. doi:10.1200/JCO.2005.04.5252

[8] K. Phillip, C. Higano, et al., "Sipuleucel-T Immunotherapy for Castration-Resistant," New England Journal of Medicine, Vol. 363, No. 5, 2010, pp. 411-422. doi:10.1056/NEJMoa1001294 PMid:20818862

[9] C. S. Higano, et al., "Integrated Data from 2 Randomized, Double-Blind, Placebo-Controlled, Phase 3 Trials of Active Cellular Immunotherapy with Sipuleucel-T in Advanced Prostate Cancer," Cancer, Vol. 115, No. 16, 2009 , 
pp. 3670-3679. doi:10.1002/cncr.24429

[10] "United States Food and Drug Administration Package Insert," 2012.

[11] R. M. Attar, C. H. Takimoto and M. M. Gottardis, "Castration-Resistant Prostate Cancer: Locking up the Molecular Escape Routes," Clinical Cancer Research, Vol. 15, No. 10, 2009, pp. 3251-3255. doi:10.1158/1078-0432.CCR-08-1171

[12] J. S. de Bono, et al., "Prednisone Plus Cabazitaxel or Mitoxantrone for Metastatic Castration-Resistant Prostate Cancer Progressing after Docetaxel Treatment: A Randomised Open-Label Trial," Lancet, Vol. 376, No. 9746, 2010, pp. 1147-1154. doi:10.1016/S0140-6736(10)61389-X

[13] “National Institutes of Health. Clinical Trials. 20," 2012. http://clinicaltrials.gov/

[14] G. Attard, et al., "Selective Blockade of Androgenic Steroid Synthesis by Novel Lyase Inhibitors as a Therapeutic Strategy for Treating Metastatic Prostate Cancer," British Journal of Urology International, Vol. 96, No. 9, 2005, pp. 1241-1246. doi:10.1111/j.1464-410X.2005.05821.x

[15] D. C. Danila, et al., "Phase II Multicenter Study of Abiraterone Acetate Plus Prednisone Therapy in Patients with Docetaxel-Treated Castration-Resistant Prostate Cancer," Journal of Clinical Oncology, Vol. 28, No. 9, 2010, pp. 1481-149. doi:10.1200/JCO.2009.25.9259

[16] J. S. de Bono, et al., "Abiraterone and Increased Survival in Metastatic Prostate Cancer," New England Journal of Medicine, Vol. 364, No. 12, 2011, pp. 1995-2005. doi:10.1056/NEJMoa1014618

[17] C. Logothetis, "Effect of Abiraterone Acetate on Pain Control and Skeletal-Related Events in Patients with Metastatic Castration-Resistant Prostate Cancer Post Docetaxel: Results from the COU-AA-301 Phase III Study," 2011.
[18] K. Fizazi, et al., "Increased Survival with Enzalutamide in Prostate Cancer after Chemotherapy," New England Journal of Medicine, Vol. 367, 2012, pp. 1187-1197.

[19] C. J. Ryan, et al., "Interim Analysis (IA) Results of COU-AA-302, a Randomized, Phase III Study of Abiraterone Acetate (AA) in Chemotherapy-Naive Patients with Metastatic Castration-Resistant Prostate Cancer (mCRPC)," Journal of Clinical Oncology, Vol. 30, 2012.

[20] F. Saad, et al., "A Randomized, Placebo-Controlled Trial of Zoledronic Acid in Patients with Hormone-Refractory Metastatic Prostate Carcinoma," Journal of National Cancer Institute, Vol. 94, No. 19, 2002, pp. 1458-1468. doi:10.1093/jnci/94.19.1458

[21] K. P. Weinfurt, et al., "Effect of Zoledronic Acid on Pain Associated with Bone Metastasis in Patients with Prostate Cancer," Annals of Oncology, Vol. 17, No. 6, 2006, pp. 286-289. doi:10.1093/annonc/md1041

[22] K. Fizazi, et al., "Denosumab versus Zoledronic Acid for Treatment of Bone Metastases in Men with CastrationResistant Prostate Cancer: A Randomised, Double-Blind Study," Lancet, Vol. 377, No. 9768, 2011, pp. 813-822. doi:10.1016/S0140-6736(10)62344-6

[23] M. Smith, et al., "Denosumab and Bone-Metastasis-Free Survival in Men with Castration-Resistant Prostate Cancer: Results of a Phase 3, Randomised, Placebo-Controlled Trial," Lancet, Vol. 379, No. 9810, 2012, pp. 39-46. doi:10.1016/S0140-6736(11)61226-9

[24] C. Parker, et al., "Updated Analysis of the Phase III, Double-Blind, Randomized, Multinational Study of Radium223 Chloride in Castration-Resistant Prostate Cancer (CRPC) Patients with Bone Metastases (ALSYMPCA)," Journal of Clinical Oncology, Vol. 30, 2012.

[25] S. Nilson, P. Strang, et al., "Bone-Targeted Radium-223 in Symptomatic, Hormone Refractory," European Journal of Cancer, Vol. 48, No. 5, 2012, pp. 678-686. 